\title{
LncRNA miR503HG Regulates the Drug Resistance of Recurrent Cervical Squamous Cell Carcinoma Cells by Regulating miR-I55/Caspase-3
}

This article was published in the following Dove Press journal: Cancer Management and Research

\section{Shanshan Zhao \\ Mingxin Yu \\ Lei Wang}

Cancer Hospital of China Medical University, Liaoning Cancer Hospital \& Institute, Gynaecology, Shenyang 1 I0042, Liaoning Province, People's Republic of China
Correspondence: Mingxin Yu Cancer Hospital of China Medical University, Liaoning Cancer Hospital \& Institute, Gynaecology, No. 44 Xiaoheyan Road, Dadong District, Shenyang II 0042 , Liaoning Province, People's Republic of China

Tel +86 I38403324I3

Email yumingxin5066@aliyun.com
Introduction: The purpose of this study is to investigate the role of long non-coding RNA (lncRNA) miR503 Host Gene (miR503HG) in cervical squamous cell carcinoma (CSCC).

Methods: Analysis of TCGA dataset revealed that expression levels of miR503HG in CSCC tissues were over 12 times lower than those in non-tumor tissues, indicating its involvement in CSCC.

Results: In this study, we observed that levels of miR503HG in plasma were significantly lower in CSCC patients than in healthy participants. The cisplatin-based treatment further downregulated miR503HG in both patients and CSCC cells. MiR503HG overexpression in CSCC cells led to the suppression of miR-155 and elevation of Caspase-3, acting as the downstream target of miR-155. Cell apoptosis analysis showed that miR503HG and Caspase-3 overexpression led to an increased cell apoptosis rate under Cisplatin treatment. MiR-155 played the opposite role and attenuated the functions of Caspase- 3 and miR503HG overexpression.

Conclusion: Therefore, miR503HG may regulate the drug resistance of CSCC cells by regulating mir-155/Caspase-3.

Keywords: cervical squamous cell carcinoma, miR503HG, miR-155, Caspase-3, apoptosis

\section{Introduction}

Cervical cancer ranks as the 4th place among all types of malignancies for both incidence and mortality rates. ${ }^{1}$ In 2018, GLOBOCAN reported that 569,847 new cases of CSCC were diagnosed, which accounts for $3.2 \%$ of all new cancer cases. ${ }^{2}$ During the same time period, 311,365 patients died due to this disease, accounting for $3.3 \%$ of all cancer mortalities. ${ }^{2}$ Surgical resection can be used to treat cervical cancer patients who were diagnosed at early stages. ${ }^{3}$ For patients diagnosed at advanced stage or recurrent CSCC patients, radiation therapies and chemotherapy are usually used. ${ }^{4,5}$ Although certain herbal extracts have also shown potentials in cancer treatment, their anti-cancer effects are limited. ${ }^{6-8}$ Cisplatin-based chemotherapy has been widely used. However, the development of chemoresistance is commonly seen, which could lead to poor survival. ${ }^{9}$

Caspase- 3 is a caspase protein that plays pivotal roles in cancer biology mainly by regulating cancer cell apoptosis. ${ }^{10}$ It has been reported that the inhibition of caspase- 3 is closely correlated with the development of drug resistance in cancer cells during chemotherapy. ${ }^{11}$ MiR-155 is a well-characterized critical factor in chemoresistance. ${ }^{12,13}$ It has been reported that miR-155 can target caspase- 3 to regulate cell apoptosis. ${ }^{14}$ In certain cases, the functions of miR-155 can be regulated by certain long non-coding 
RNAs (lncRNAs, >200nt). ${ }^{15}$ LncRNA MIR503 Host Gene (miR503HG) has been reported to be a tumor suppressor in several types of cancers. ${ }^{16,17}$ However, its exact functioning role in cervical cancer is unclear. By analyzing the TCGA dataset, we found that miR503HG was significantly downregulated in cervical squamous cell carcinoma (CSCC), which is a major subtype of cervical cancer. This study is aimed to investigate the roles of miR503HG in CSCC, as well as its possible interactions with miR-155 and Caspase-3.

\section{Materials and Methods}

\section{CSCC Patients}

The present study enrolled 46 cases of CSCC patients (females, 39-69 years' old, 53.6 \pm 6.1 years' old) from the 133 cases of CSCC admitted to Cancer Hospital of China Medical University between March 2015 and March 2019. The review board of the aforementioned hospital Ethics Committee approved this study. Inclusion criteria: 1) recurrent or advanced stages (III or IV) CSCC can be treated by chemotherapy; 2) no other severe complications were observed. Exclusion criteria: 1) chemotherapy received within 3 months before admission; 2) patients with expected survival time shorter than 3 months. During the same time period, 46 cases of healthy participants (females, $39-69$ years, $54.2 \pm 6.3$ years) were also selected from the routine physical health examination center of the aforementioned hospital to serve as controls. Age and gender distributions were not significantly different between CSCC and control groups. All participants were informed of the experimental design. Informed consent was signed by all patients. This study was conducted in accordance with the Declaration of Helsinki.

\section{Treatment and Sampling}

On the day of admission (before any therapies), blood $(5 \mathrm{~mL})$ was extracted from each participant under a fasting condition. The 46 CSCC patients were treated with cisplatin at a dosage of $50 \mathrm{mg} / \mathrm{m}^{2}, 3$ weeks per time for varied time (at least 9 weeks). Blood was also collected from each patient under fasting conditions at 3, 6 and 9 weeks after treatment. All blood samples were centrifuged in EDTA tubes for $15 \mathrm{~min}$ at $1200 \mathrm{~g}$ to prepare plasma.

\section{CSCC Cell Lines and Transient Transfections} C33A and SiHa human CSCC cell lines (ATCC, USA) were used as CSCC cell model. Cell culture medium contained $10 \%$ FBS and 90\% Eagle's Minimum Essential Medium.
Cells were cultivated in a $5 \% \mathrm{CO}_{2}$ incubator at $37{ }^{\circ} \mathrm{C}$ with $95 \%$ humidity. Cells were harvested at $70-80 \%$ confluence to perform subsequent transfections.

Vectors expressing miR503HG and Caspase-3 were constructed using the pcDNA3.1 vector (Sangon, Shanghai, China). Negative control (NC) miRNA and miR-155 mimic were the products of Sangon (Shanghai, China). Lipofectamine 2000 (Sangon) was used to transfect $10 \mathrm{nM}$ vectors (empty vector as $\mathrm{NC}$ group) or $50 \mathrm{nM}$ miRNA (NC miRNA as NC group) into $10^{6}$ cells. All operations were completed according to the manufacturer's instructions. All the following experiments were performed at $24 \mathrm{~h}$ posttransfection.

\section{RNA Extractions and qPCR}

RNAzol (Sigma-Aldrich, USA) was used to extract RNAs from $10^{5} \mathrm{C} 33 \mathrm{~A}$ or $\mathrm{SiHa}$ cells as well as $0.4 \mathrm{~mL}$ plasma. In cases of cisplatin treatment, cells were cultivated in cell culture medium containing $50 \mu \mathrm{M}$ cisplatin for $24 \mathrm{~h}$ and $48 \mathrm{~h}$ before use. Control cells were cultivated in normal mediums at the same time. To harvest miRNAs, $85 \%$ of ethanol was used to precipitate RNAs. Genomic DNAs in RNA samples were removed by performing DNase I digestion.

PrimeScript RT Reagent Kit (TaKaRa, Japan) was used to perform total RNA reverse transcriptions with poly (T) as a primer to prepare cDNA samples. With cDNA as a template, all qPCR reactions were prepared using SYBR Green Master Mix (Bio-Rad) to measure the expression levels of miR503HG and Caspase-3. In this assay, GAPDH was used as an endogenous control.

The levels of mature miR-155 expression were measured by using All-in-One ${ }^{\mathrm{TM}}$ miRNA qRT-PCR Detection Kit from GeneCopoeia (Guangzhou, China). In this assay, U6 was used as the endogenous control to measure the expression levels of miR-155.

\section{Cell Apoptosis Assay}

$\mathrm{C} 33 \mathrm{~A}$ and $\mathrm{SiHa}$ cells were harvested at $24 \mathrm{~h}$ posttransfection. Cell pellets containing $10^{5}$ cells were resuspended $1 \mathrm{~mL}$ cell culture medium containing $50 \mu \mathrm{M}$ cisplatin. Cells were cultivated under the aforementioned cell culture conditions for $48 \mathrm{~h}$. After that, $0.25 \%$ trypsin digestion was performed and Annexin V-FITC as well as propidium iodide staining was followed. Finally, apoptotic cells were separated using flow cytometry. 


\section{Western Blot}

RIPA solution (Sangon) was used to extract total proteins from C33A and SiHa cells harvested at $24 \mathrm{~h}$ post-transfection. All protein samples were denatured in boiling water for $10 \mathrm{~min}$. To separate proteins, $10 \%$ SDS-PAGE gel was used to carry out electrophoresis. Following gel transfer to PVDF membranes, blocking was performed at room temperature for $2 \mathrm{~h}$ in PBS containing $5 \%$ non-fat milk. Following that, Caspase 3 (ab4051, 1:2000, Abcam) and GAPDH (ab9485, 1:2000, Abcam) rabbit polyclonal primary antibodies were used to incubate with the membranes for 18 at $4^{\circ} \mathrm{C}$. After that, membranes were further incubated with $\mathrm{HRP}$ (IgG) secondary antibody (goat anti-rabbit, ab97051, 1:2000, Abcam) for $2 \mathrm{~h}$ at room temperature. ECL (Sigma-Aldrich, USA) was used to produce signals. All data normalizations were performed using Image J v.1.46.

\section{Statistical Analysis}

Three biological replicates were included in each experiment and mean values were used to perform data analysis. Comparisons among multiple groups or between two groups were performed by one-way ANOVA in combination with the Tukey's test and unpaired $t$-test, respectively. Comparisons among different time points in the same group were performed by repeated-measures ANOVA. $\mathrm{p}<0.05$ was statistically significant.

\section{Results}

\section{Downregulation of miR503HG Was Observed in CSCC}

Differential expression of miR503HG in CSCC was first analyzed using TCGA dataset. It was observed that the expression level of miR503HG was more than 12 times lower in CSCC tissues than in non-cancer tissues (2.2 vs 27.6). To further confirm its downregulation, qPCR was used to measure levels of miR503HG in plasma from both CSCC patients $(n=46)$ and healthy participants $(n=46)$. Expression data were compared between two groups by unpaired $t$-test. It was observed that plasma levels of miR503HG were also significantly lower in CSCC patients than in healthy participants (Figure 1, $\mathrm{p}<0.05$ ).

\section{Cisplatin Treatment Further Downregulated miR503HG in Both Patients and CSCC Cells}

Expression levels of miR503HG in plasma collected from CSCC patients at 0,3,6 and 9 weeks after the initiation of Cisplatin treatment were measured by qPCR. Comparisons

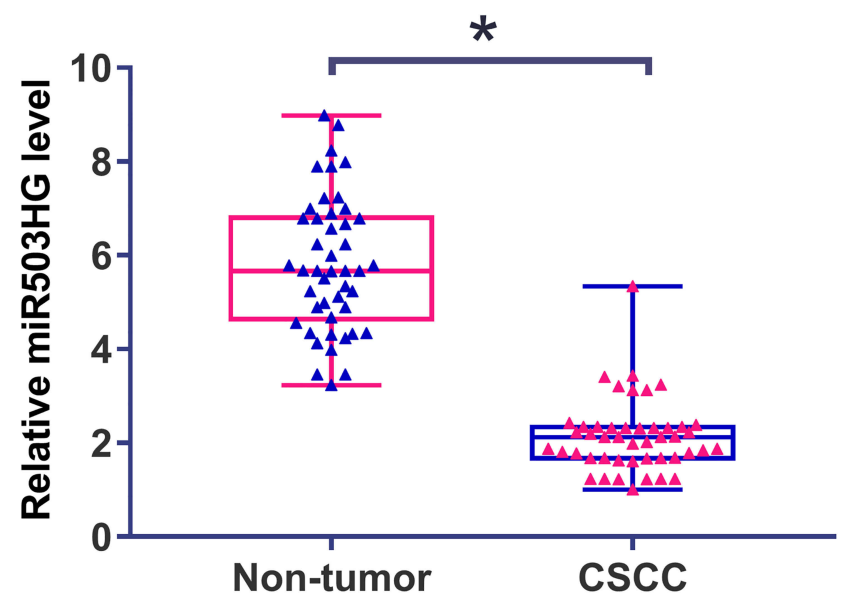

Figure I Downregulation of miR503HG was observed in CSCC. qPCR was used to measure levels of miR503HG in plasma from both CSCC patients $(n=46)$ and healthy participants $(n=46)$. Expression data were compared between the two groups by unpaired $t$-test. qPCR was repeated 3 times and mean values were presented $(* p<0.05)$.

among different time points were performed by repeatedmeasures ANOVA. It was observed that Cisplatin treatment led to significantly downregulated plasma levels of miR503HG in a time-dependent manner (Figure 2A, $\mathrm{p}<0.05)$. C33A and SiHa cells were cultivated in cell culture medium containing $50 \mu \mathrm{M}$ cisplatin for $24 \mathrm{~h}$ and $48 \mathrm{~h}$, followed by the measurement of miR503HG expression level by performing qPCR. Control cells were cultivated in normal medium for the same time. It was observed that Cisplatin treatment led to significantly downregulated miR503HG in both $\mathrm{C} 33 \mathrm{~A}$ and $\mathrm{SiHa}$ cells in a timedependent manner (Figure 2B, $\mathrm{p}<0.05$ ). In addition, Cisplatin treatment o led to significantly upregulated miR155 (Supplemental Figure 1A) and significantly downregulated Caspase-3 mRNA (Supplemental Figure 1B) in both $\mathrm{C} 33 \mathrm{~A}$ and SiHa cells in a time-dependent manner $(\mathrm{p}<0.05)$.

\section{Overexpression of miR503HG Altered miR-I55 and Caspase-3 Expression}

C33A and SiHa cells were transfected with a miR503HG expression vector. Overexpression of miR503HG was confirmed by $\mathrm{qPCR}$ at $24 \mathrm{~h}$ post-transfection. Comparing to C (untransfected cells) and NC (cells transfected with empty vector) groups, expression levels of miR503HG were significantly increased at $24 \mathrm{~h}$ post-transfection (Figure $3 \mathrm{~A}, \mathrm{p}<0.05$ ). Moreover, miR503HG overexpression in CSCC cells led to the downregulation of miR-155 (Figure $3 \mathrm{~B}, \mathrm{p}<0.05$ ). In contrast, miR503HG overexpression led to increased expression levels of Caspase-3 at both mRNA (Figure 3C) and protein (Figure 3D) levels $(\mathrm{p}<0.05)$. 

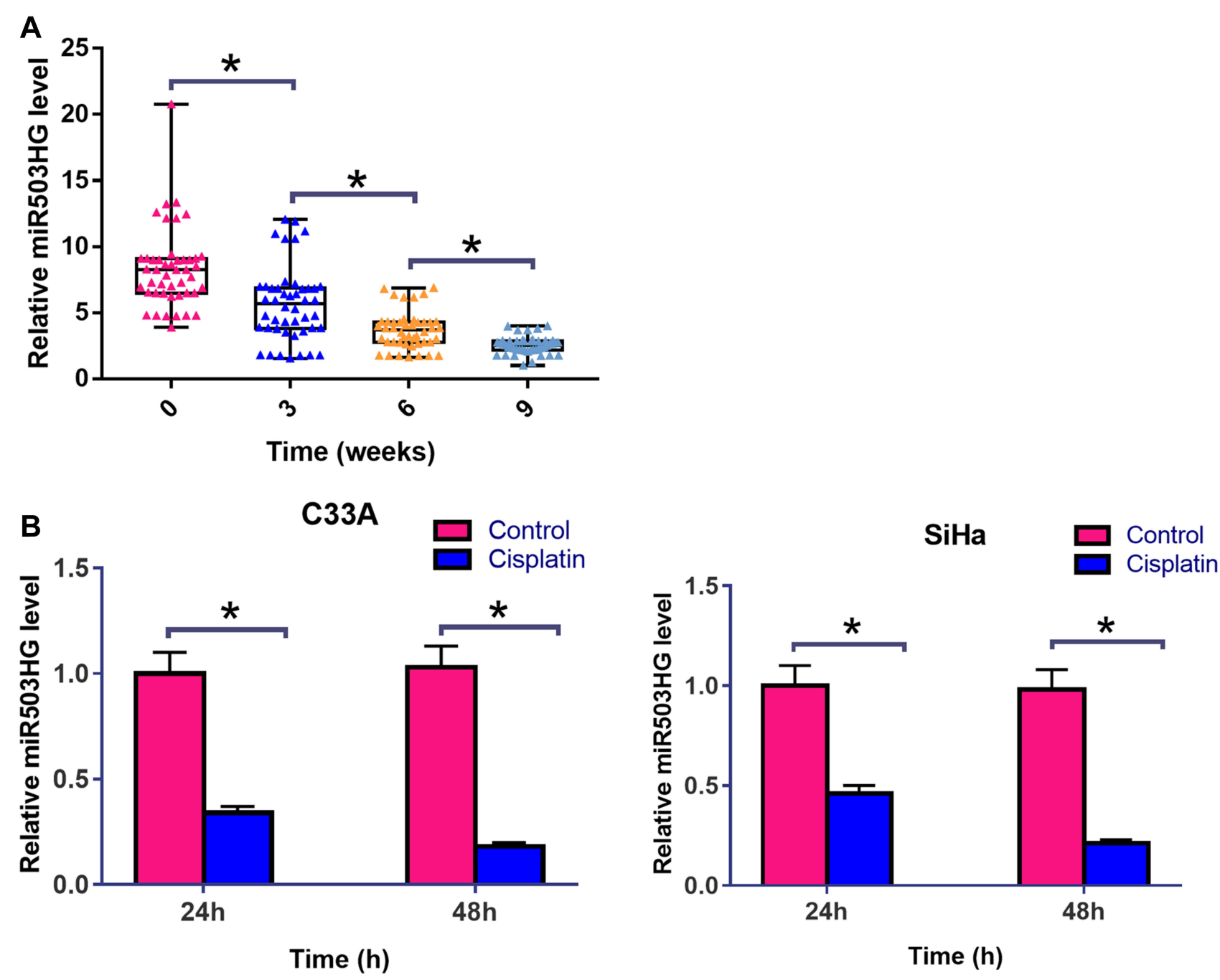

Figure 2 Cisplatin treatment further downregulated miR503HG in both patients and CSCC cells. Expression levels of miR503HG in plasma collected from CSCC patients at $0,3,6$ and 9 weeks after the initiation of cisplatin treatment were measured by qPCR. Comparisons among different time points were performed by repeated measures ANOVA (A). $\mathrm{C} 33 \mathrm{~A}$ and $\mathrm{SiH}$ cells were cultivated in cell culture medium containing $50 \mu \mathrm{M}$ cisplatin for $24 \mathrm{~h}$ and $48 \mathrm{~h}$, followed by the measurement of miR503HG expression level by performing qPCR. control cells were cultivated in normal medium for the same time. Comparisons between control and cisplatin groups were performed by unpaired $t$-test (B). qPCR reactions were repeated 3 times. Data were expressed as mean values $\left({ }^{*} \mathrm{p}<0.05\right)$.

\section{MiR503HG Regulated CSCC Apoptosis Through miR-I55 and Caspase-3}

Cell apoptosis assay was performed to analyze the overexpression of miR503HG, miR-155, and Caspase- 3 on the apoptosis of $\mathrm{C} 33 \mathrm{~A}$ and $\mathrm{SiHa}$ cells. Compared with $\mathrm{C}$ and NC (empty vector or NC miRNA) groups, miR503HG, and Caspase-3 overexpression led to an increased cell apoptosis rate. MiR-155 played the opposite role and attenuated the effects of Caspase- 3 and miR503HG overexpression (Figure 4, $\mathrm{p}<0.05$ ).

\section{Discussion}

In this study, we reported the tumor-suppressive roles of miR503HG in CSCC. Our data showed that miR503HG was downregulated in CSCC and was further inhibited during cisplatin treatment. We also showed that miR503HG may promote CSCC apoptosis through miR-155 and Caspase-3 during cisplatin treatment.

The tumor-suppressive functions of miR503HG have been reported in several types of cancer. In hepatocellular carcinoma (HCC), miR503HG was downregulated and its overexpression can alter HNRNPA2B1/NF- $\mathrm{BB}$ signaling to suppress tumor metastasis. ${ }^{16}$ We analyzed the TCGA dataset and we also confirmed the downregulation of miR503HG in HCC than in non-tumor tissues (0.55 vs.2.1). In bladder cancer, miR503HG was also downregulated and suppressed cancer progression by inhibiting cancer cell proliferation and invasion. ${ }^{17}$ Similarly, we also 


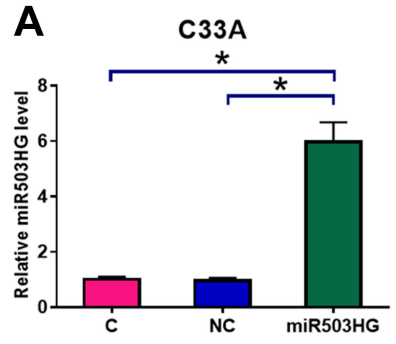

C

C33A

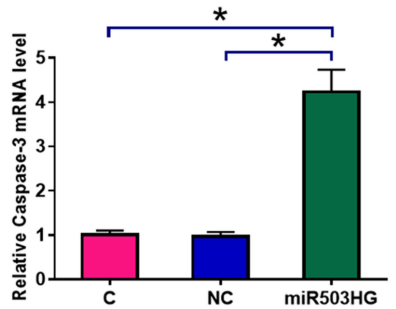

$\mathrm{SiHa}$

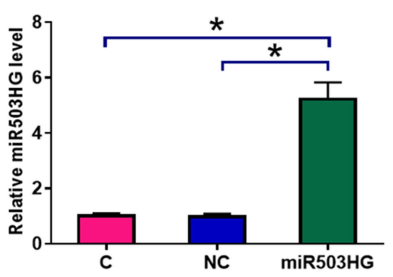

D
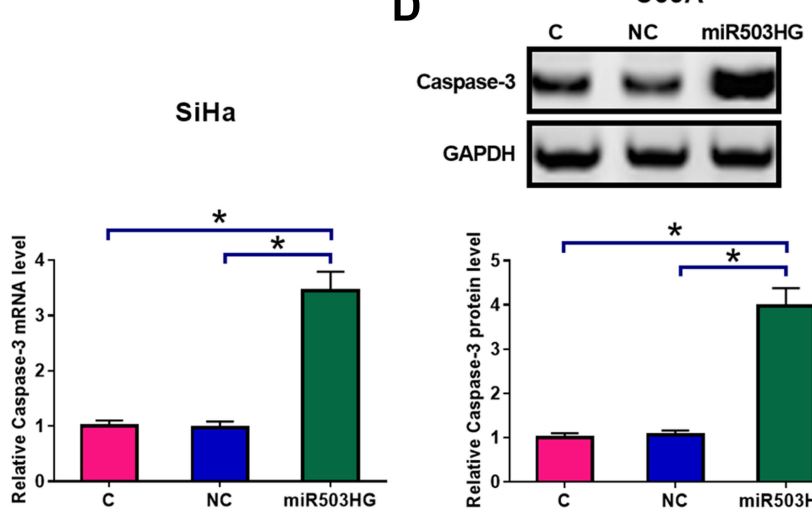

B
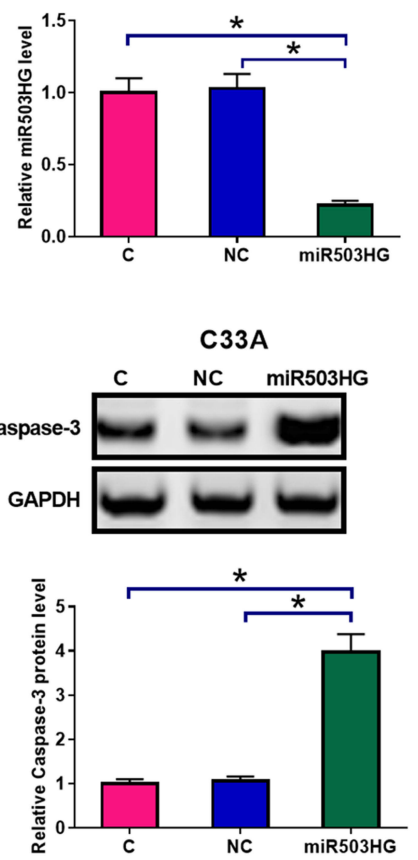

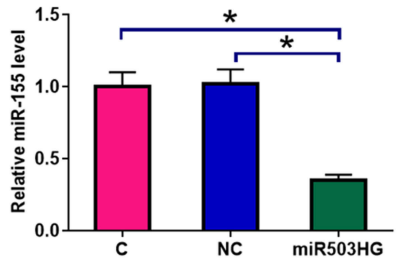

$\mathrm{SiHa}$
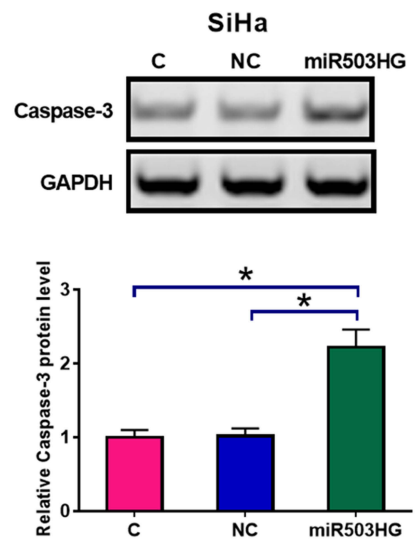

Figure 3 Overexpression of miR503HG led to altered miR-155 and caspase-3 expression. C33A and SiHa cells were transfected with a miR503HG expression vector. Overexpression of miR503HG was confirmed by qPCR at $24 \mathrm{~h}$ post-transfection (A). qPCR results showed that miR503HG overexpression in CSCC cells led to the downregulation of miR-155 (B). In contrast, qPCR and Western blot experiments showed that miR503HG overexpression led to increased expression levels of caspase-3 at both mRNA $(\mathbf{C})$ and protein $(\mathbf{D})$ levels. Experiments were repeated 3 times and mean values were presented $\left({ }^{*} \mathrm{p}<0.05\right)$.

\section{C33A}

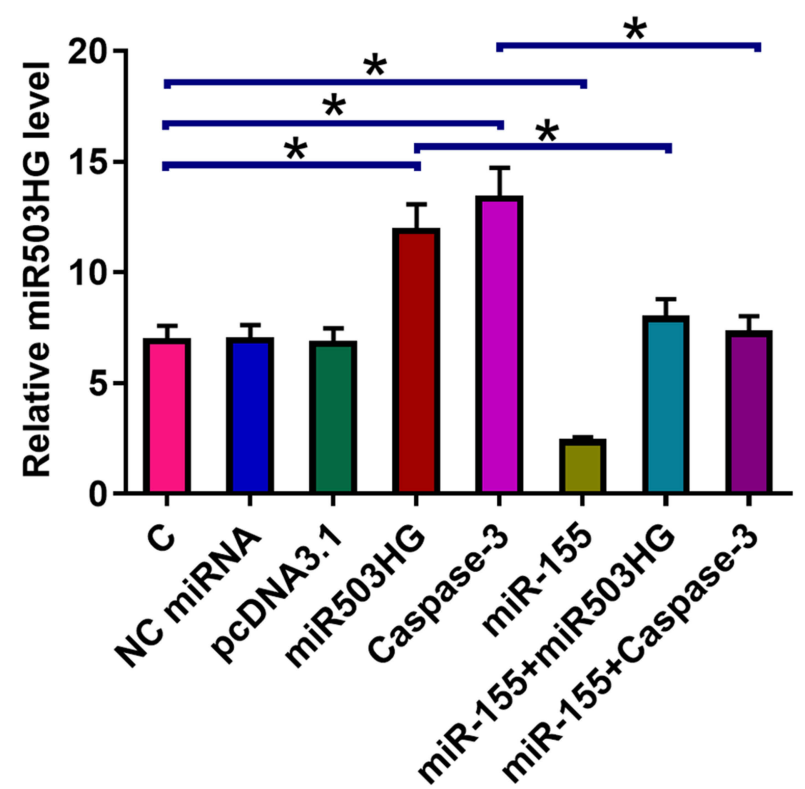

\section{$\mathrm{SiHa}$}

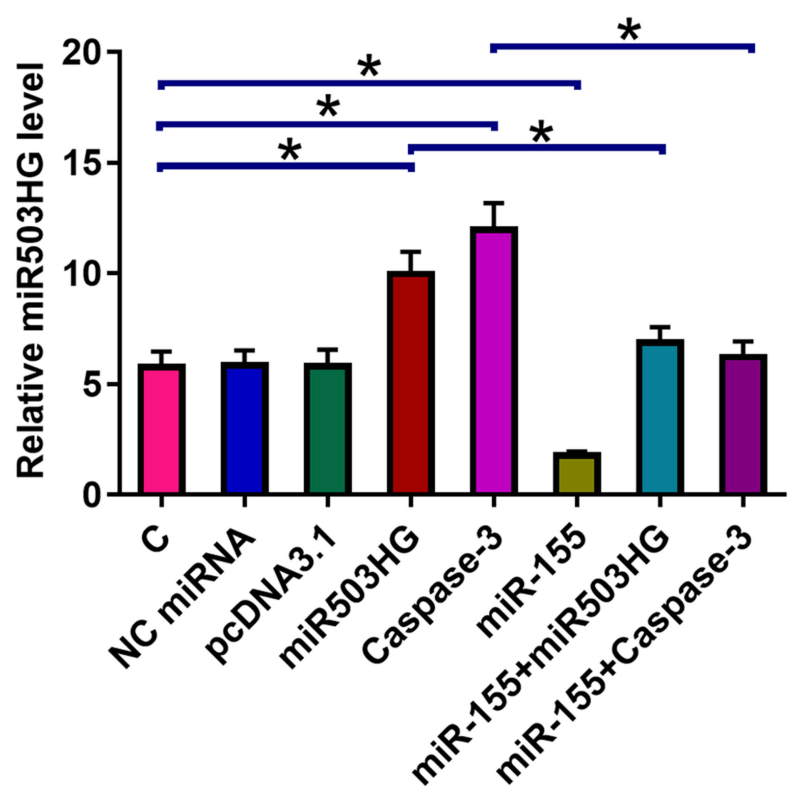

Figure 4 MiR503HG regulated CSCC apoptosis through miR-I55 and Caspase-3. Cell apoptosis assay was performed to analyze the overexpression of miR503HG, miR155, and Caspase-3 on the apoptosis of $\mathrm{C} 33 \mathrm{~A}$ and $\mathrm{SiHa}$ cells. Experiments were repeated 3 times and mean values were presented $\left({ }^{*} \mathrm{p}<0.05\right)$.

observed the downregulation of miR503HG in bladder cancer in the TCGA dataset (1.75 vs 2.25). However, the downregulation of miR503HG in these two types of cancer is less than 4-fold. In our study, we observed a more than 12-fold downregulation of miR503HG in CSCC of TCGA dataset. We also confirmed the downregulation of 
miR503HG in plasma from CSCC patients. Therefore, miR503HG is likely a tumor suppressor in CSCC.

It has been well established that miR-155C has important roles in the modulation of cisplatin sensitivity. ${ }^{18}$ Caspase- 3 is a direct target of miR-155. In this study, we observed downregulated miR-155 and upregulated Caspase-3 after miR503HG overexpression. In addition, miR503HG promoted the apoptosis of CSCC cells under cisplatin treatment. It has been well established that Caspase-3 plays critical role in mediating cisplatin-induced cancer cell apoptosis. ${ }^{19}$ Our cotransfection experiments also showed that miR-155 may mediate the interaction between Caspase-3 and miR503HG to participate in cisplatin-induced CSCC cell apoptosis. Therefore, a novel miR503HG, miR-155 and Caspase-3 pathway involved in the regulation of the sensitivity of CSCC cells to cisplatin was characterized in this study.

In this study, we also observed the downregulation of miR503HG in both CSCC patients and cell lines after cisplatin treatment. Previous studies showed that lncRNAs are critical factors in cisplatin-resistant cervical cancer cells. ${ }^{20,21}$ Therefore, miR503HG is likely a downstream effector of cisplatin. In view of our observation that miR503HG is downregulated in CSCC patients who received cisplatin treatment overexpression of miR503HG may serve as a target to improve cisplatin sensitivity. However, the mechanism of the regulation of miR-155 by miR503HG is unknown. Our future studies will perform deeper investigations. The downregulation of plasma miR503HG may also serve as a biomarker to guide the determination of the dosage of cisplatin and the use of other therapies.

In conclusion, miR503HG was downregulated in CSCC and may regulate miR-155 and Caspase-3 to modulate the sensitivity of CSCC cells to cisplatin.

\section{Disclosure}

The authors report no conflicts of interest in this work.

\section{References}

1. Small W Jr, Bacon MA, Bajaj A, et al. Cervical cancer: a global health crisis. Cancer. 2017;123(13):2404-2412. doi:10.1002/cncr.v123.13

2. Bray F, Ferlay J, Soerjomataram I, et al. Global cancer statistics 2018: GLOBOCAN estimates of incidence and mortality worldwide for 36 cancers in 185 countries. CA Cancer J Clin. 2018;68(6):394-424. doi:10.3322/caac. 21492

3. Bentivegna E, Gouy S, Maulard A, et al. Oncological outcomes after fertility-sparing surgery for cervical cancer: a systematic review. Lancet Oncol. 2016;17(6):e240-e253. doi:10.1016/S1470-2045(16)30032-8

4. Lutgens LCHW, Koper PCM, Jobsen JJ, et al. Radiation therapy combined with hyperthermia versus cisplatin for locally advanced cervical cancer: results of the randomized RADCHOC trial. Radiother Oncol. 2016;120(3):378-382. doi:10.1016/j.radonc.2016.02.010
5. Tsuda N, Watari H, Ushijima K. Chemotherapy and molecular targeting therapy for recurrent cervical cancer. Chin J Cancer Res. 2016;28(2):241-253. doi:10.21147/j.issn.1000-9604.2016. 02.14

6. Wang J, Zhang YS, Thakur K, et al. Licochalcone A from licorice root, an inhibitor of human hepatoma cell growth via induction of cell apoptosis and cell cycle arrest. J Agric Food Chem. 2018; 120:407-417.

7. Zhang YY, Zhang F, Zhang YS, et al. Mechanism of juglone-induced cell cycle arrest and apoptosis in Ishikawa human endometrial cancer cells. J Agric Food Chem. 2019;67(26):7378-7389. doi:10.1021/acs. jafc. $9 \mathrm{~b} 02759$

8. Wang J, Liao AM, Thakur K, et al. Licochalcone B extracted from glycyrrhiza uralensis fisch induces apoptotic effects in human hepatoma cell HepG2. J Agric Food Chem. 2019;67(12):3341-3353. doi:10.1021/acs.jafc.9b00324

9. Zhu H, Luo H, Zhang W, et al. Molecular mechanisms of cisplatin resistance in cervical cancer. Drug Des Devel Ther. 2016;10:1885-1895. doi:10.2147/DDDT.S106412

10. Chung CW, Song YH, Kim IK, et al. Proapoptotic effects of tau cleavage product generated by caspase-3. Neurobiol Dis. 2001;8 (1):162-172. doi:10.1006/nbdi.2000.0335

11. Devarajan E, Sahin AA, Chen JS, et al. Down-regulation of caspase 3 in breast cancer: a possible mechanism for chemoresistance. Oncogene. 2002;21(57):8843-8851. doi:10.1038/sj.onc. 1206044

12. Bayraktar R, Van Roosbroeck K. miR-155 in cancer drug resistance and as target for miRNA-based therapeutics. Cancer Metastasis Rev. 2018;37(1):33-44. doi:10.1007/s10555-017-9724-7

13. Van Roosbroeck K, Fanini F, Setoyama T, et al. Combining anti-miR155 with chemotherapy for the treatment of lung cancers. Clin Cancer Res. 2017;23(11):2891-2904. doi:10.1158/1078-0432.CCR16-1025

14. Wang HQ, Yu XD, Liu ZH, et al. Deregulated miR-155 promotes Fas-mediated apoptosis in human intervertebral disc degeneration by targeting FADD and caspase-3. J Pathol. 2011;225(2):232-242. doi:10.1002/path.2931

15. Yan K, Fu Y, Zhu N, et al. Repression of lncRNA NEAT1 enhances the antitumor activity of $\mathrm{CD} 8+\mathrm{T}$ cells against hepatocellular carcinoma via regulating miR-155/Tim-3. Int $J$ Biochem Cell Biol. 2019;110:1-8. doi:10.1016/j.biocel.2019.01.019

16. Wang $\mathrm{H}$, Liang L, Dong Q, et al. Long noncoding RNA miR503HG, a prognostic indicator, inhibits tumor metastasis by regulating the HNRNPA2B1/NF- $\mathrm{BB}$ pathway in hepatocellular carcinoma. Theranostics. 2018;8(10):2814-2829. doi:10.7150/thno. 23012

17. Qiu F, Zhang M, Zhou Z, et al. IncRNA MIR503HG functioned as a tumor suppressor and inhibited cell proliferation, metastasis and epithelial-mesenchymal transition in bladder cancer. $J$ Cell Biochem. 2019;120(6):10821-10829. doi:10.1002/jcb.28373

18. Pouliot LM, Chen YC, Bai J, et al. Cisplatin sensitivity mediated by WEE1 and CHK1 is mediated by miR-155 and the miR-15 family. Cancer Res. 2012;72(22):5945-5955. doi:10.1158/0008-5472.CAN$12-1400$

19. Arjumand W, Seth A, Sultana S. Rutin attenuates cisplatin induced renal inflammation and apoptosis by reducing NFкB, TNF- $\alpha$ and caspase-3 expression in wistar rats. Food Chem Toxicol. 2011;49 (9):2013-2021. doi:10.1016/j.fct.2011.05.012

20. Wang B, Huang Z, Gao R, et al. Expression of long noncoding RNA urothelial cancer associated 1 promotes cisplatin resistance in cervical cancer. Cancer Biother Radiopharm. 2017;32(3):101-110. doi:10.1089/cbr.2016.2156

21. Chi C, Mao M, Shen Z, et al. HOXD-AS1 exerts oncogenic functions and promotes chemoresistance in cisplatin-resistant cervical cancer cells. Hum Gene Ther. 2018;29(12):1438-1448. doi:10.1089/hum. 2017.256 


\section{Publish your work in this journal}

Cancer Management and Research is an international, peer-reviewed open access journal focusing on cancer research and the optimal use of preventative and integrated treatment interventions to achieve improved outcomes, enhanced survival and quality of life for the cancer patient.

The manuscript management system is completely online and includes a very quick and fair peer-review system, which is all easy to use. Visit http://www.dovepress.com/testimonials.php to read real quotes from published authors.

Submit your manuscript here: https://www.dovepress.com/cancer-management-and-research-journal 